Cahiers d'études africaines

\title{
Sociologie actuelle des funérailles
}

Prise en charge de la mort et espace social dans l'Afrique d'aujourd'hui

Current Sociology of Funerals. The Management of Death and the Social Space in Contemporary Africa

\section{Joël Noret}

\section{OpenEdition \\ Journals}

\section{Édition électronique}

URL : http://journals.openedition.org/etudesafricaines/21633

DOI : $10.4000 /$ etudesafricaines. 21633

ISSN : 1777-5353

\section{Éditeur}

Éditions de l'EHESS

\section{Édition imprimée}

Date de publication : 1 décembre 2017

Pagination : 1011-1033

ISBN : 978-2-7132-2687-8

ISSN : 0008-0055

Référence électronique

Joël Noret, «Sociologie actuelle des funérailles », Cahiers d'études africaines [En ligne], 228 | 2017, mis en ligne le 01 décembre 2019, consulté le 03 janvier 2020. URL : http://journals.openedition.org/ etudesafricaines/21633; DOI : 10.4000/etudesafricaines.21633 


\title{
Sociologie actuelle des funérailles
}

\author{
Prise en charge de la mort et espace social \\ dans l'Afrique d'aujourd'hui
}

\begin{abstract}
Les funérailles africaines ont toujours exprimé les rapports sociaux et les relations de pouvoir autant que les croyances : dramatisation où la société se donne à voir et à vivre dans un moment de forte intensité (Balandier 1991 : 7).
\end{abstract}

L'objectif de ce texte est double. Il s'agit d'abord de revenir sur la rencontre largement manquée entre d'une part les travaux portant sur le changement social africain - dans lesquels va s'inscrire Balandier à partir des années 1950 - et d'autre part la dynamique des funérailles africaines de la même période. Il s'agit ensuite de montrer brièvement, à partir des recherches que je mène au Bénin depuis plus de quinze ans, l'actualité de l'héritage intellectuel de Balandier et d'une sociologie du changement social pour travailler sur les funérailles africaines d'aujourd'hui.

Ce texte revient donc d'abord sur une relative absence, celle des funérailles, dans la sociologie des modernités africaines dans laquelle Balandier s'engage en France essentiellement à partir des années 1950. Cette absence relative se déploie en effet sur fond d'une double présence. Car d'une part, les funérailles sont déjà bien présentes à l'époque dans la littérature de sciences sociales sur les sociétés non européennes - elles tiennent par exemple une place significative dans les architectures théoriques d'un Durkheim ou d'un Van Gennep. D'autre part, les funérailles africaines sont marquées, au milieu du $\mathrm{XX}^{\mathrm{e}}$ siècle, par des transformations importantes, liées en particulier à l'urbanisation et au changement religieux. Balandier est le témoin direct de ces évolutions. Pour autant, en dépit de cette double présence des funérailles, théorique et empirique, celles-ci ne laisseront que peu de traces dans les travaux de Balandier comme dans ceux d'ailleurs des sciences sociales du changement social africain qui se mettent en place dans le deuxième tiers du $\mathrm{XX}^{\mathrm{e}}$ siècle. 
Pourtant, partir à la recherche des évocations de funérailles dans le travail du Balandier africaniste, c'est voir se dessiner par petites touches une anthropologie politique de celles-ci et de leurs dynamiques qui ne verra véritablement le jour dans la littérature africaniste que bien plus tard, à partir des années 1980. La seconde partie de ce texte reviendra dès lors sur certains aspects des funérailles du Bénin méridional d'aujourd'hui à partir de cet héritage intellectuel, qui en restitue notamment la dimension « d'économodrames » (Balandier 1982 [1955] : 510) révélateurs de divisions sociales multidimensionnelles. Car travailler avec Balandier aujourd'hui, c'est notamment se souvenir de son attention à la dynamique et à l'hétérogénéité du social et remettre sur le métier l'éclectisme méthodologique bien tempéré à partir duquel il construit ses travaux africanistes.

\section{Funérailles et changement social : retour sur une rencontre manquée}

\section{Un objet spectral}

Partons d'un triple constat. Premièrement, il faut se souvenir ici que la mort et les rites funéraires figurent en bonne place à l'agenda théorique de l'anthropologie depuis les débuts de la discipline. Sans même remonter aux travaux évolutionnistes de Tylor ou Frazer (Palgi \& Abramovitch 1984), nombre d'auteurs classiques ont consacré à la question de la mort des pages importantes. Les funérailles sont ainsi l'un des rites de passage essentiels à partir desquels Van Gennep (1981 [1909]) construit et étaie ses propositions. Dans les travaux de l'École française de sociologie, Hertz (1970 [1907]) et Durkheim (1994 [1912]) lui-même consacrent de nombreuses pages aux relations entre la prise en charge de la mort et la vie des collectifs, d'où les funérailles ressortent comme des moments fondamentaux de la restauration des groupes sociaux, une perspective prolongée par Radcliffe-Brown (1922), et par Malinowski (1954 [1925]).

Deuxièmement, c'est en fait dès le tournant des années 1930 que l'anthropologie va commencer à se confronter, sur les terrains africains, à la question du changement social. Les articles fondateurs à cet égard de Malinowski, et son offre de services à l'administration coloniale, sont bien connus (de L'Estoile 1994). Malinowski (1935 : 480-481) suggère en effet dès les années 1930 que l'objet central de l'anthropologie est le "changing native », regrettant de ne pas avoir pris acte plus tôt de ce qui lui apparaît alors comme un état de fait ${ }^{1}$. Dans les décennies 1930 et 1940 , les textes sur le « contact

1. On notera au passage l'écart avec la problématique annoncée moins de quinze ans auparavant dans l'Introduction aux Argonautes, où l'objet de l'anthropologie était conçu comme 
culturel » (Malinowski 1929, 1945 ; Mair 1934) vont se succéder, puis être passés au crible de la critique gluckmanienne et notamment de son insistance à rapatrier pleinement la situation coloniale - et plus largement l'histoire et les rapports sociaux - dans la construction de l'objet (Gluckman 1940, 1947). Au tournant des années 1950, quand Balandier fait son entrée dans le champ, l'étude du changement social a donc déjà une certaine profondeur et constitue un objet bien identifié, dont l'actualité ne va faire que se renforcer dans le climat de l'Après-guerre et l'inflexion des politiques coloniales auquel il correspond. Les années 1950 verront se multiplier les textes prenant à bras le corps les transformations des sociétés africaines. Tantôt en renvoyant explicitement à la question du changement social - le sous-titre original de Sociologie actuelle de l'Afrique noire (Balandier 1982 [1955]) est à cet égard éloquent ${ }^{2}$. Tantôt en déployant de nouvelles problématiques qui apparaissent alors au cœur des transformations en cours - il faut penser le développement du phénomène urbain, les transformations du monde du travail, les nouvelles formes de différenciation sociale et les formes inédites d'organisation sociale, l'évolution des structures villageoises elles-mêmes sous l'effet de la situation coloniale, ou encore le changement religieux et le déploiement des christianismes africains indépendants des structures missionnaires.

Troisièmement, dans les travaux de plus en plus nombreux qui, dans les décennies 1950 et 1960, font émerger une anthropologie et une sociologie du changement social africain, les funérailles apparaissent plutôt incidemment, parfois au détour d'une page, en particulier lorsqu'il est question de la vie associative qui se développe alors rapidement dans les nouveaux foyers urbains de l'Afrique coloniale. Dans un article programmatique portant sur l'étude du changement social en Afrique de l'Ouest britannique, Little (1953) souligne ainsi au détour d'une page l'aide mutuelle que se portent les membres des associations urbaines à l'occasion des funérailles. Ottenberg (1955) fait des remarques analogues sur les associations qui se développent alors dans le monde rural en transformation du pays ibo, dans le sud-est du Nigeria. Mitchell (1956: 19) consacre deux brefs passages de son Kalela Dance à évoquer combien les " sociétés de funérailles» ("burial societies») sont au cœur du phénomène associatif dans certaines villes de Rhodésie du Nord comme du Sud, quoique de manière inégale selon les agglomérations, et comment les transformations de l'ethnicité dans la « situation urbaine» - l'une de ses thèses centrales — se donnent aussi à voir à l'occasion des funérailles (ibid. : 29). Dans la même veine d'allusions brèves à l'articulation entre nouvelles associations urbaines et funérailles, Balandier note lui

l'étude de «l'humanité sauvage» (MALINOWSKI 1984 [1922]).

2. «La dynamique des changements sociaux en Afrique centrale». 
aussi rapidement la perception de cotisations et «l'entr'aide très mesurée » qu' assurent les « associations d'originaires » à l'occasion des deuils dans les Brazzavilles noires, ainsi que la rôle significatif que peuvent prendre certaines associations de jeunes femmes faisant preuve d'une «liberté sexuelle affirmée » dans les funérailles de leurs membres, dont les cérémonies de levée de deuil prennent alors place dans des bars (Balandier 1985a [1955] : 122-123, 146-147 ; Tonda 2000) $)^{3}$.

On pourrait certainement multiplier les exemples. Mais ce qui se dégage de ce corpus de littérature alors en plein développement, c'est une focalisation — pour l'essentiel — sur les questions sociales, politiques et économiques qui apparaissent alors comme les plus pressantes, sans intérêt soutenu pour — ni véritable détour par - les phénomènes qui pourraient apparaître comme secondaires dans le grand récit de la modernisation qui hante ces travaux à des degrés divers — grand récit avec lequel Balandier (1985b [1974] : 141-152) eut par ailleurs assez tôt l'occasion de prendre une certaine distance. Les orientations thématiques et les urgences théoriques sont autres. Les funérailles restent ici un objet spectral, d'arrière-plan, qui resurgit çà et là au fil des pages, sans véritablement recevoir une attention et une analyse spécifiques. Ainsi, la fréquentation des ouvrages du Balandier des décennies 1950 à 1970 suggère que l'intérêt des funérailles pour une sociologie ou une anthropologie dynamiste ou dynamique passe plutôt inaperçu.

On croise bien encore dans Afrique ambiguë le décès accidentel d'un orpailleur et les rites de purification qui s'ensuivent à proximité de Bamako, ou un cortège funèbre transportant le cercueil d'un enfant à Brazzaville, mais ce sont là des situations évoquées en une demi-page (ibid. 2008 [1957]: 93, 269). Dans le même volume, la cérémonie de clôture de deuil d'un chef pygmée babinga reçoit davantage d'attention, car elle est l'occasion de manœuvres politiques entre chefs d'ethnies voisines pour le contrôle du produit de la chasse des Pygmées (ibid. : 186-198). Dans Sociologie actuelle enfin (Balandier 1982 [1955]), une partie de l'intérêt de Balandier pour les « dynamiques sociales totales » se déploie à partir de la description de fêtes données notamment à l'occasion des levées de deuil d'hommes importants,

3. On trouve aussi, à l'époque, des textes qui, sans problématiser à proprement parler la question des évolutions des funérailles, en prennent acte au passage tout en développant un autre propos. C'est le cas par exemple dans les « Nyakyusa Conventions of Burial» de G. WILSON (1939), dont le projet théorique est de souligner l'importance des affects et des sentiments dans la vie sociale, mais où G. WILSON $(1939$ : 10-12, 24) note également au passage l'affaissement partiel de l'attachement aux — et de l'investissement dans les rites funéraires «traditionnels » parmi les jeunes générations en particulier, sous l'effet des missions chrétiennes présentes en pays nyakyusa depuis le tournant du $\mathrm{XX}^{\mathrm{e}}$ siècle, et l'émergence d'obsèques chrétiennes. 
fêtes qu'il s'excuse, au moment d'y revenir en conclusion, de n'avoir que « trop rapidement évoquées » (ibid. : 503) - les malaki. Ceux-ci sont donnés périodiquement par l'homme fort d'un lignage dans une logique d'entretien et de production des alliances lignagères. Ils impliquent la réception et le divertissement d'alliés, ainsi que des échanges de dons, certains de ceux-ci prenant au milieu du $\mathrm{XX}^{\mathrm{e}}$ siècle une forme monétaire. Le malaki se donne ainsi à voir comme «manifestation d'alliance et défi économique » (ibid. : 384), la cérémonie festive pouvant aussi être l'occasion lors de laquelle se règle « la transmission des biens et la dévolution des charges » (ibid. : 507), tout au moins lorsque le malaki correspond à une levée de deuil. Car on n'est pas ici à proprement parler sur la scène des funérailles — les malaki peuvent aussi se tenir indépendamment de levées de deuil, et il existe des funérailles sans malaki. Mais Balandier s'efforce toutefois clairement dans ces pages de penser des dynamiques sociales cruciales à partir d'une approche de la vie rituelle pleinement attentive à ses enjeux politiques et économiques. Se trouve alors tracée la voie d'une anthropologie politique des funérailles qui scrute les relations d'alliance et de concurrence entre « grands hommes » et entre groupes. Anthropologie politique des funérailles à laquelle il faudra encore une trentaine d'années pour être pleinement mobilisée dans le champ des études africaines.

\section{La genèse tardive d'une sociologie dynamique des funérailles}

Moments de mise en jeu de rapports sociaux multidimensionnels, les funérailles auraient en fait très bien pu être constituées en «révélateur» à part entière de dynamiques cruciales de la période coloniale. Car le temps colonial fut bel et bien, dans les mondes urbains qui se développaient comme dans une partie au moins des mondes ruraux africains, un moment de transformations significatives des formes de prise en charge de la mort, lesquelles auraient parfaitement pu nourrir une anthropologie ou une sociologie «dynamique ». C'est ce que montreront toute une série de travaux historiques menés rétrospectivement. Une lecture attentive de la littérature qui émerge dès les années 1950 sur les associations permet d'ailleurs de voir les funérailles se loger au cœur de l'émergence du phénomène associatif africain pendant la période coloniale. C'est de là notamment que partira Terence Ranger, en s'intéressant aux sociétés d'assistance funéraire lorsque son attention se portera sur l'histoire des funérailles dans l'Afrique australe urbaine de la première moitié du $\mathrm{XX}^{\mathrm{e}}$ siècle. Ainsi, Ranger (2004) a pu montrer qu'à Bulawayo, dans le poumon industriel de la Rhodésie du Sud, les funérailles avaient constitué un site de 
lutte symbolique tout à fait important pendant la période coloniale. Au cœur des townships de l'Afrique australe, dans ce qui pourrait apparaître comme un monde quadrillé par le pouvoir colonial, son travail montre que de nombreuses initiatives émanant du nouveau monde urbain ont bel et bien fait émerger une culture funéraire dynamique dont l'enjeu central était de « dignifier la mort». Ranger explore ainsi la naissance et l'activité des sociétés d'entraide funéraire (burial societies), les rites funéraires mis en place par les Églises indépendantes « éthiopiennes » ou " apostoliques », les rites funéraires traditionnels transposés dans les cimetières urbains, ou encore les rapatriements de corps vers les régions rurales — « il y a eu une politique de la mort dans la Bulawayo noire » (ibid. : 135), écrit-il on ne peut plus clairement dans une formule dont la traduction en français évoque inévitablement les Brazzavilles noires.

Les travaux historiques des deux dernières décennies en particulier ont ainsi souligné, dans différentes parties du continent, les transformations - les mutations, aurait écrit Balandier (1970) à une certaine époque — des funérailles africaines pendant le temps colonial, marquées à la fois par des contraintes structurelles et des aspirations nouvelles, se reflétant notamment dans le changement religieux et d'autres formes de créativité culturelle. On pourrait ici aussi multiplier les exemples (Parker 2000 ; Jindra 2005 ; Onwuzuruigbo 2014). Mais pour n'évoquer qu'une autre situation marquante, les travaux d'Yvan Droz (2011) ou de Mark Lamont (2011) sur les hauts-plateaux du centre du Kenya explorent le basculement du mode de disposition des corps qui s'accélère à partir des années 1930, sous la triple poussée des préoccupations hygiénistes de l'administration coloniale, de pressions foncières et du christianisme missionnaire, relayé ici par de nouvelles élites locales scolarisées et christianisées. Dans le centre du Kenya en effet, le mode dominant de disposition des cadavres était, jusqu'au cœur de la période coloniale, l'abandon aux charognards, seuls certaines personnes âgées remplissant historiquement les conditions pour être inhumées. Mais la possibilité de faire reconnaître des droits sur le foncier à partir de la présence de tombes dans un contexte de concurrence foncière accrue, le souci sanitaire enchâssé dans le projet civilisateur de l'État colonial et les progrès du christianisme missionnaire, nourrirent de différentes manières un ensemble de préoccupations portées en particulier par des représentants des jeunes générations scolarisées et christianisées, menant finalement à une transformation radicale des formes de ritualisation de la mort dans les populations meru et kikuyu, la décennie 1930 étant ici celle du véritable basculement (Droz 2011 ; Lamont 2011).

Dans une part significative de l'Afrique au sud du Sahara, les funérailles sont aujourd'hui bien connues pour être des arènes sociales majeures, où se font et se défont hiérarchies et relations de patronage, mais aussi alliances et 
solidarités diverses, en bref les collectifs et les divisions sociales ${ }^{4}$. Or, si la recherche contemporaine fait désormais une large place à la dynamique des funérailles et à leur importance dans les processus de recomposition sociale en cours, une telle perspective n'a réellement commencé à émerger que dans les années 1980 (Vidal 1986 ; Gilbert 1988). Pendant les décennies qui sont celles de l'émergence d'une sociologie et d'une anthropologie du changement social africain en effet, les rites funéraires sont restés largement envisagés dans les contextes ruraux et au prisme des ritualités « ethniques 》 et « traditionnelles 》 (notamment Morton-Williams 1960 ; Fortes 1961; Goody 1962; Jackson 1977 ; Middleton 1982).

Dans certains travaux, conceptions de la mort et rites funéraires étaient même, d'une certaine façon, l'un des grands lieux anthropologiques de production de l'altérité africaine. Un lieu de la tradition par excellence. En France, on pense notamment aux travaux emblématiques sur ce point de Louis-Vincent Thomas (1995: 64), lequel se lamentait encore à la fin de sa vie des « assauts destructeurs » de la modernité sur les rites funéraires de "l'Afrique noire traditionnelle » - la filiation post-griaulienne dans laquelle Thomas (1960, 2006) s'inscrivait à certains égards n'ayant pas empêché par ailleurs l'installation d'une forme de complicité entre Balandier et lui à la Sorbonne, pour ce qui est de leurs rapports personnels 5 . Et ceci fait aussi écho à la double Afrique de Balandier, chez lequel André Mary (2017) décèle justement une « tension entre l'ethnologue de l'Afrique des traditions "immémoriales" et le sociologue du changement social et de la modernité africaine».

En 1986 toutefois, Claudine Vidal traite véritablement les funérailles en « révélateur » des dynamiques de la société ivoirienne (abidjanaise) de l'époque. Les funérailles, écrit-elle, $\mathrm{y}$ " institutionnalisent une mise en scène générale des relations de pouvoir et de dépendance » (Vidal 1986:9). L'investissement des acteurs y est total, tout à la fois psychique, social et économique, car aucun ne peut se dérober à ce jeu social profondément ancré dans l'illusio locale, « sociodrame » passionné où tenir son rang acquiert une dimension existentielle. Le ton est donné par les dépenses somptuaires de la «bourgeoisie d'État». Tandis que dans les classes populaires, on subit « avec une anxiété résignée la survenue de cette catastrophe funéraire à répétition » (ibid. : 10). Des associations constituées sur la base des origines régionales ou villageoises assurent régulièrement la perception de cotisations que nul ne peut véritablement

4. On pourrait ici multiplier les références, qui se sont accumulées dans les deux dernières décennies en particulier. Une revue de la littérature reprenant l'essentiel des travaux publiés en anglais et en français (à l'exception malheureuse de COHEN \& ODHIAMBo [1992]) peut être trouvée dans JINDRA \& NORET (2011).

5. Communication personnelle de C. Javeau, courrier électronique du 8 juin 2017. 
esquiver sous peine d'un déclassement social radical. Car la valeur de chacun s'éprouve ici « par rapport aux autres, devant et contre les autres » (ibid. : 13). En un mot, les « sociodrames funéraires » sont ici traités en occasions majeures de théâtralisation des "rapports de domination », et pleinement constitués en objet digne d'une anthropologie ou d'une sociologie politique. Inscrivant son travail dans le sillage des perspectives ouvertes en France par Balandier ${ }^{6}$, Vidal lui demande d'ailleurs quelques années plus tard une préface - citée en exergue de cet article - à Sociologie des passions, volume où sera repris «Funérailles et conflit social», enrichi d'un second texte dans une section baptisée «L'amour du village». L'introduction au volume renvoie cette fois explicitement à la notion d'économodrame forgée par Balandier notamment à partir des malaki, et dont tout laisse à penser qu'elle a inspiré le recours de Vidal (1991: 9-11) à l'idée de sociodrame.

Placés dans le sillage de l'idée maussienne de «prestations totales », les «économodrames» de Balandier (1982 [1955] : 503) sont en effet des moments de "consommation massive de richesses ». Ainsi, les "fêtes publiques» périodiques fang ( bilaba ») ou kongo («malaki») dans lesquelles notables, chefs ou groupes lignagers dans leur ensemble rivalisent de générosité constituent autant d'occasions de « dépense » au cours desquelles le capital économique d'hommes importants est converti en capital social et symbolique, dans une économie profondément marquée par l'impératif de la production d'alliances. Moments de «"dramatisation" des rapports économiques et sociaux», ces événements cérémoniels rendent explicites "des relations sociales fondamentales » et « offrent au regard une sorte de "résumé" du tout social » (ibid. : 509). On l'a vu, ce n'est pas seulement ni directement chez Balandier de funérailles qu'il s'agit, même si certains de ces événements peuvent correspondre à des levées de deuil. Mais c'est probablement lorsqu'il envisage ces prestations totales et ces faits cérémoniels dans leur multidimensionnalité, et en particulier comme phénomènes économiques et politiques, que Balandier se rapproche le plus d'une perspective sur les funérailles qui les constituerait pleinement en «révélateurs » - ce qui constitue au fond le geste de Vidal trente ans après Sociologie actuelle.

Dans la seconde partie de ce texte, je voudrais à présent glisser mes pas dans ceux des économodrames de Balandier et des sociodrames de Vidal pour montrer qu'une entrée par les funérailles peut se révéler sociologiquement productive lorsqu'il s'agit d'explorer les lignes de division de la société béninoise d'aujourd'hui. Mais aussi que l'éclectisme méthodologique bien tempéré du Balandier des Brazzavilles noires, associant enquête de terrain, travail en archives et données de cadrage d'ordre quantitatif, n'a certainement

6. Communication personnelle de Claudine Vidal, courrier électronique du 6 juin 2017. 
pas perdu de son actualité ni de sa pertinence pour ressaisir sous des angles complémentaires la complexité des dynamiques sociales africaines.

\section{Funérailles et divisions sociales dans le Bénin méridional}

\section{La production d'un ordre funéraire}

En deux mots, la différenciation, sociale et religieuse, de la société du Bénin méridional s'est accrue, en particulier à partir du XIX ${ }^{e}$ siècle, avec l'établissement progressif de marchands «afro-brésiliens» sur ce qui était alors la "Côte des Esclaves », et l'émergence puis le développement de minorités musulmanes puis catholiques. Elle s'est accélérée au XXe siècle avec le fait colonial et les dynamiques qu'il engagea ou auxquelles il contribua, dans tous les domaines de la vie sociale. Ainsi, pour ce qui est des funérailles, si les lignages restent souvent, aujourd'hui encore, au cœur de la prise en charge de la mort et de l'organisation des scénarios funéraires, l'exercice de l'autorité en leur sein à ces occasions n'est plus reconnu aussi facilement qu'auparavant à leurs chefs coutumiers. Les acteurs sociaux sont également, en particulier en milieu urbain, de plus en plus engagés dans des mondes sociaux différents et ce, dans un contexte marqué notamment par la dispersion géographique grandissante des familles, par des inégalités économiques importantes, par une stratification éducative socialement saillante, ainsi que par la montée en puissance et la priorité sociale croissante des identités religieuses.

Un paradoxe des funérailles contemporaines veut ainsi que, si le cadre lignager a perdu beaucoup de son évidence dans la vie quotidienne de bien des Béninois, les funérailles sont incontestablement un moment où il faut compter avec l'avis des autorités de son lignage, et ce en dépit de la distance sociale qui peut parfois s'être installée. Car la différenciation sociale travaille bien évidemment les collectivités lignagères de l'intérieur, et ce sont donc régulièrement des individus issus de mondes sociaux différents qui doivent s'entendre sur une trame de funérailles. En effet, les enfants ne décident pour ainsi dire jamais seuls, et le scénario des funérailles s'élabore pas à pas dans le cadre de «réunions familiales» où un consensus est recherché entre les options privilégiées par les enfants (qui se sont régulièrement concertés en amont), leurs oncles et tantes, et les autorités lignagères, à savoir les hommes désignés au sein du lignage pour en administrer la vie collective et les intérêts. C'est donc entre ces groupes, lorsqu'ils cherchent à privilégier des options divergentes quant au canevas des funérailles, que peuvent naître des conflits 
qui se résoudront soit par l'effacement ou la soumission de certaines parties, soit par la formation d'un compromis, fût-il minimal (Noret 2010, 2014).

En effet, les discussions entourant l'organisation de l'enterrement, le montant des cotisations, la répartition des dépenses, le choix de la date, du lieu et de l'ordre rituel des cérémonies, peuvent s'avérer des occasions de tensions, voire de conflits, non négligeables, par exemple entre partisans d'un enterrement exclusivement chrétien et partisans du cumul des registres rituels chrétien et traditionaliste. Ou entre tenants d'une inhumation au cimetière et tenants d'une mise en terre dans la concession lignagère du défunt. Et ce, sans même évoquer les questions d'héritage qui peuvent déjà se profiler à cette occasion. Ainsi, une recherche menée il y a une petite dizaine d'années sur les budgets ménagers dans deux quartiers populaires de Cotonou, et incluant des questions sur les dépenses de protection de type magico-religieux, a-t-elle pu faire apparaître un recouvrement significatif entre ceux ayant eu à faire face à des dépenses de funérailles importantes et ceux ayant recouru à des services de protection magico-religieuse. Et l'implication économique dans des funérailles fait augmenter de manière significative la probabilité d'avoir à consentir également des dépenses de protection magico-religieuse (Lemay, Noret \& Somville 2013). C'est que les funérailles sont bien des moments de tractations et de frictions lors desquels se fait particulièrement ressentir le besoin de se protéger, et en particulier de se prémunir de potentielles manœuvres occultes en provenance des parties avec lesquelles des désaccords ont vu ou pourraient voir le jour. Un tel constat corrobore d'ailleurs assez étroitement l'enquête ethnographique, qui suggère notamment que les funérailles sont des moments d'insécurité économique, sociale et psychique, à l'occasion desquels il faut - en particulier pour les enfants et beaux-enfants du mort, sur lesquels pèse l'essentiel de la charge financière - pouvoir mobiliser des ressources allant en général bien au-delà de ses moyens, se montrer à la hauteur du rang social qui est le sien, afin d'être finalement confirmé à la fois dans son statut social et, pour les enfants, dans l'idée qu'on est un enfant digne du défunt (Noret 2012). La réception organisée après l'inhumation à l'attention de l'assistance, voire du quartier, « révèle » à cet égard des aspects essentiels des funérailles, en même temps que des dynamiques plus larges de l'espace social béninois, livrant par certains aspects, pour reprendre le mot de Balandier, « une sorte de "résumé" du tout social».

En effet, si l'idée de « résumé » du social peut être discutée, la réception est certainement l'épisode des funérailles contemporaines qui en est le plus visible, et le plus investi. Elle mobilise ainsi le plus largement le capital social des endeuillés, et accapare la part la plus importante de leur investissement économique. La réception qui suit la mise en terre n'a toutefois pas 
toujours eu les contours qui sont les siens depuis une vingtaine d'années. Sa forme actuelle, qui la voit prendre place immédiatement après l'inhumation, est en fait étroitement dépendante du développement du recours aux morgues pour une période de plus en plus longue, un phénomène qui concerne aujourd'hui de nombreux pays côtiers en Afrique de l'Ouest (Noret 2004; van der Geest 2006 ; Page 2007), et qui a bouleversé la grammaire temporelle des funérailles. Dans le Bénin méridional d'il y a un demi-siècle en effet, les corps étaient enterrés rapidement, et l'essentiel des investissements se concentraient sur des moments ultérieurs du cycle funéraire, couplés le cas échéant à une messe de commémoration (Noret 2006). Aujourd'hui, la mobilisation la plus massive des réseaux sociaux et des ressources prend indéniablement place autour de la cérémonie d'inhumation elle-même. C'est là une transformation qui s'est d'abord amorcée dans les classes supérieures, historiquement davantage citadines et catholiques que le reste de la société. L'examen des annonces nécrologiques révèle par exemple très clairement que le développement de la pratique du dépôt des corps à la morgue — aujourd'hui banalisée pour les défunts d'un âge suffisamment avancé pour avoir des enfants adultes - a d'abord concerné les hommes (et dans une moindre mesure, les femmes) âgés des classes supérieures. Dans la première moitié des années 1980, on voit en effet s'allonger progressivement le délai entre le décès et l'inhumation dans le monde social des élites urbaines, qui sont alors seules à publier des annonces nécrologiques dans le quotidien national de la République populaire ${ }^{7}$. Les catholiques réguliers ayant droit à une «messe corps présent » pour leurs obsèques (signe qu'en principe ils pratiquaient et communiaient régulièrement jusqu'à leur mort) sont alors surreprésentés, ainsi que les milieux afro-brésiliens (ibid.). Dans le Bénin contemporain comme dans la Côte d'Ivoire des années 1980 qu'évoque Claudine Vidal, ce sont désormais ces classes supérieures qui donnent le ton et dictent les critères de «réussite» des funérailles, au cœur desquelles la réception a pris une place centrale. Mais place maintenant à l'ethnographie, au travers de deux courtes vignettes.

7. Consultation des mois de juillet, août et septembre 1979 du quotidien Ehuzu, quotidien officiel de l'État sous le régime d'inspiration marxiste-léniniste, aux Archives nationales de Porto-Novo. Consultation des mois de janvier, février, mars, juillet et août $1981 \mathrm{du}$ quotidien Ehuzu aux Archives nationales de Porto-Novo. Consultation des mois de janvier, février et mars 1985 du quotidien Ehuzu aux Archives nationales de Porto-Novo (NORET 2006). 
Scènes de funérailles

Scène 1. Après la messe d'inhumation de la mère de David à Abomey, en février 2004, le cortège funèbre prit d'abord la route de la concession lignagère du père de la défunte, où celle-ci devait être enterrée ${ }^{8}$. Une partie de l'assistance suivit le corbillard, tandis que d'autres groupes se dirigeaient directement vers la concession lignagère du mari de la défunte, à quelques centaines de mètres, où se tiendrait la réception. Des chaises avaient ici été disposées sous quelques bâches dans deux cours de la concession, ainsi que contre les murs le long desquels le rebord d'un toit forme une bande d'ombre. La plus grande partie de la réception était organisée dans ces deux cours, de façon assez peu hiérarchisée et compartimentée, car on n'était pas ici dans une situation où la défunte avait plusieurs enfants organisant des réceptions séparées. Les deux enfants avaient ici organisé le gros de la réception ensemble. David, gendarme de son état, avait toutefois prévu un endroit à l'écart où recevoir ses hôtes de marque, et en particulier ses collègues, tandis que sa sœur, une femme illettrée, séparée depuis longtemps de son mari et sans véritable capital économique, recevait ses plus proches parents et amis dans le salon de la maison construite par David dans la concession lignagère, attenante à l'une des deux cours, et dont elle habite une partie au quotidien.

Arrivant pour ma part après l'inhumation, accompagné de mon assistant de recherche, nous nous asseyons contre un mur ombragé de la cour, où nous sommes servis une première fois. Mais la fille de la défunte nous remarque et nous demande de nous déplacer plutôt dans le salon de la maison, où elle reçoit les invités qu'elle veut distinguer. Elle n'a pas prévu de menus différents selon les catégories de membres de l'assistance, mais une minorité d'hôtes " de marque ", comme on dit volontiers localement, sont néanmoins reçus à l'intérieur, avec des portions plus richement garnies et accompagnées de davantage de bouteilles de bière ou de sodas que les invités reçus à l'extérieur. Là, nous sommes servis une deuxième fois. Mais David, circulant dans la réception pour s'assurer de son bon déroulement, et venu comme il se doit saluer et échanger des politesses avec les invités en train de manger, nous remarque à son tour, et nous invite alors à le suivre dans le bâtiment, situé un peu à l'écart, où il reçoit ses invités personnels. Nous ressortons de la concession et y rentrons par une autre porte, une vingtaine de mètres plus loin. Là, dans une petite cour et le salon d'une maison en construction, à l'écart de la foule qui se presse dans la partie centrale de la concession lignagère, une bonne dizaine de gendarmes et quelques autres invités sont assis et mangent

8. Je reprends dans cette première vignette, sous une forme réécrite et condensée, des passages d'une étude de cas déployée dans NorEt (2010). 
en plaisantant. On nous sert d'emblée un whisky de marque, puis une forme de cassoulet caractéristique des menus de funérailles des couches moyennes et supérieures de la population - soit un menu et des boissons qui n'étaient pas proposés hors de cet espace réservé. Rassasiés, nous refuserons les autres plats qu'on se presse de nous servir... Déplacé à deux reprises au cours de la réception, par des hôtes qui cherchaient chaque fois à me mettre à une place qui nous honore et nous distingue mutuellement, j'ai aussi littéralement traversé ce jour-là l'espace (à la fois social et physique) des positions dans lequel les invités d'une réception sont répartis. D'abord assis avec les membres de l'assistance la plus commune, faite de parents éloignés et d'habitants du quartier, j'ai été amené deux fois de suite au cœur du dispositif prévu par chacun des enfants pour recevoir ses invités privilégiés. Participant alors déjà depuis l'avant-veille aux cérémonies «traditionnelles » ayant précédé la messe d'inhumation, j'avais eu l'occasion, en amont de la réception, de remettre aux deux enfants endeuillés une enveloppe de soutien.

Scène 2. Après l'inhumation de la mère d'Honoré au cimetière de Ouidah, au début de juillet 2005, après six semaines de préparations pendant lesquelles le corps avait été laissé aux bons soins de la morgue de la ville, ce cadre de l'État avait prévu de recevoir ses invités dans une institution publique de la ville dont il était le directeur. Pratiquant ordinaire de l'appropriation privative du lieu, il avait réparti les différentes catégories d'invités, selon le type de relation (familiale, professionnelle, etc.) et selon leur statut, dans différentes pièces des bâtiments et dans la cour. Quelques jeunes, actifs dans l'institution ou redevables à Honoré d'une manière ou d'une autre, orientaient les invités. Pour « assister » ainsi Honoré, celles et ceux qui le pouvaient avaient en plus acheté le tissu choisi comme «uniforme» des funérailles. Occupés à préparer la réception depuis le matin, ils s'apprêtaient maintenant à servir les invités. Peut-être Honoré, plus tard, leur revaudrait-il leur dévouement. Mais il leur aurait surtout difficilement pardonné leur indisponibilité pour le service. Chacun(e) savait qu'il ne goûterait pas une telle farce, et était dès lors présent à son poste.

Les membres de la « famille » étaient invités à s'asseoir sous deux bâches dressées dans la cour. Les membres de la presse, collègues d'Honoré qui intervenait aussi régulièrement sur les ondes d'une radio locale, étaient orientés vers une partie de la salle des fêtes de l'institution. Enfin, les « autorités » étaient orientées vers la pièce jouxtant immédiatement le bureau du directeur. Les « autorités » forment une catégorie sociale cruciale dans le Bénin méridional, qui renvoie le plus souvent aux cadres du public et du privé, et de manière plus générale aux personnes auxquelles leur réussite économique assure un certain pouvoir d'influence. Lors des funérailles de la mère d'Honoré, 
étaient essentiellement orientés vers cette pièce des «autorités », les cadres et directeurs locaux des secteurs de l'enseignement et de la culture, secteurs dans lesquels Hervé était un notable local. Bonne connaissance d'Honoré depuis déjà quelques années à ce moment-là, c'est avec ces « autorités » que j'avais été placé.

Les différentes catégories d'invités étaient placées à distance suffisante les unes des autres pour ne pas savoir vraiment ce qui était servi aux autres, c'est-à-dire comment les autres étaient « reçus ». Mais c'est bien dans la pièce où je me trouvais alors que les alcools les plus coûteux seraient servis, les plats garnis le plus généreusement en viande ou en poisson, la bière distribuée sans compter, et quelques bouteilles de vin posées sur les tables. Le menu du jour comptait in fine une potée de légumes agrémentée de morceaux de saucisse de Francfort, des quarts de poulet braisés avec un féculent au choix, et de la viande de chèvre braisée pour clôturer. Soit des mets choisis et relativement coûteux, de la viande plutôt que du poisson, et des boissons servies à volonté, dans un lieu réservé et non exposé à la vue du tout-venant - en un mot, un cadre et un menu conformes à ce que pouvaient être les attentes de distinction des « autorités» ayant fait le déplacement.

Après avoir mangé, mes deux voisins dirent leur appréciation pour le service. La réception était déjà en train de finir pour nous. Je me levai et cherchai à voir Honoré pour le saluer avant de partir. Je l'ai trouvé dans son bureau, occupé à discuter avec l'un de ses invités en piquant des morceaux de viande braisée dans une assiette. Il m'a demandé si tout s'était bien passé, et je l'ai rassuré en lui certifiant, comme cela se fait, que nous avions été très bien servis, et en ajoutant que je n'avais entendu que des commentaires positifs autour de moi, ce qui était d'ailleurs le cas. Comme il s'apprêtait à retourner voir ses invités, je l'ai accompagné dehors et, une fois dans la cour, lui ai discrètement remis l'enveloppe que j'avais préparée pour «l'assister », avant de prendre congé.

\section{L'espace des positions au prisme de la réception}

La réception est un moment crucial des funérailles. Elle est d'abord le moment qui draine la foule la plus massive - tout le monde ne fait pas le déplacement pour la cérémonie d'inhumation, et encore moins vers le cimetière ou la pièce réservée pour inhumer le défunt. Mais surtout, elle représente de loin le poste de dépenses le plus important, focalisant par ailleurs à ce titre une part significative de l'anxiété des endeuillés (Noret 2012). Quelques centaines de personnes transitent régulièrement par une réception de funérailles, sinon 
davantage, auxquelles il faut assurer le boire et le manger. Les budgets de réception varient énormément selon les couches de la population et ce, à l'image des inégalités de revenus, considérables dans la région. Ainsi, dans les milieux défavorisés, on peut difficilement investir plus d'une centaine de milliers de francs CFA (150 €) dans une réception. Mais le coût des réceptions organisées par les couches moyennes et supérieures de la population peut facilement atteindre plusieurs centaines de milliers de francs CFA, et les commerçants établis et les cadres organisent des réceptions dont le coût dépasse régulièrement un ou deux millions de francs CFA ( 1500 ou $3000 €$ ), voire bien davantage pour ce qui est des "grands commerçants», des cadres supérieurs, des hommes d'affaires les plus en vue ou des personnes connectées au sommet de l'État.

Une partie de la réception est souvent organisée en commun par les enfants et beaux-enfants du défunt - sur lesquels repose l'essentiel des dépenses - mais chacun(e) aura aussi à cœur d'organiser une réception "privée », mieux approvisionnée, pour recevoir parents plus proches et connaissances. Dans les couches moyennes et supérieures de la population, on prévoit souvent ici différents «niveaux» de réception selon le type de relation et le statut des invités : on ne recevra pas ses collègues cadres avec ses oncles «villageois", sa belle-famille avec les membres de son groupe de prières, etc. Fonctionnaires et autres cols blancs, ainsi que commerçants ayant une certaine surface économique, se reposent en outre aujourd'hui de plus en plus massivement sur des « services-traiteurs » pour leurs réceptions de funérailles, lesquels leur garantissent un service de niveau supérieur à celui des groupes de femmes auxquels on peut recourir dans chaque quartier pour le même type d'occasions.

Ainsi, si «dans un premier temps », selon le mot de Bourdieu (1984:3), « la sociologie se présente comme une topologie sociale » cherchant à cartographier le monde social, la réception renvoie au sociologue l'image d'un exercice de sociologie profane, où le sens local des divisions sociales s'exerce à travers l'organisation d'un espace (physique) où il s'avère essentiel pour les organisateurs de placer chacun selon son rang. L'exercice peut se révéler relativement délicat, en raison en particulier du caractère multidimensionnel des positions et des divisions sociales, phénomène valable dans toutes les sociétés, mais dont Balandier (2004 [1971] : 267-299) a souligné avec force la saillance dans les sociétés africaines postcoloniales, où coexistent «plusieurs modes de stratification ». Un point éloquemment souligné aussi par Claudine Vidal (1986: 15-16), qui évoquait déjà la difficulté de l'entreprise dans la société ivoirienne d'il y a une trentaine d'années : «Il s'agit de réunir des fractions sociales très inégales et les susceptibilités en matière d'étiquette 
s'avivent en ces moments où n'ont rien de fixe les compromis entre les règles coutumières de respect et l'observance des actuelles distances sociales. »

$\mathrm{Au}$ sens du placement des organisateurs de la réception répond en outre le sentiment que peuvent avoir de leur place les différentes composantes de l'assistance. Car comme l'illustrent ci-dessus les commentaires d'appréciation du service que mes voisins échangeaient à l'issue de la réception de l'enterrement de la mère d'Honoré, la réception est le premier moment d'évaluation des funérailles par l'assistance. Les endeuillés sont ainsi toujours attentifs à placer leurs hôtes au mieux, en veillant tout particulièrement à ce qu'on ne leur reproche pas d'avoir «minimisé » quelqu'un, comme on dit localement. Chacun doit avoir été reçu selon son «niveau », un enjeu important pour les endeuillés étant d'éviter que ne coure après les funérailles le bruit selon lequel les choses n'auraient pas été faites "proprement».

Chacun se souvient de cas fumeux, et les bruits qui courent sur des réceptions mal organisées n'honorent pas les familles concernées. L'attention portée à ses « invités » pendant la réception, puis les échanges de politesses, les félicitations, mais aussi les bénédictions des hôtes sur le départ («vous avez bien fait», "votre papa [défunt] vous bénira », etc.) sont donc des moments importants, où les uns remercient d'avoir été bien reçus et soulignent la réussite des funérailles, et les autres remercient leurs invités d'avoir fait le déplacement, leur demandent conventionnellement si leurs remerciements et leurs félicitations sont sincères, cherchent tout aussi conventionnellement à retenir encore un peu leurs invités, leur proposent encore à boire ou à manger - rares sont en fait les endeuillés qui prennent le temps de s'asseoir au cours des réceptions qu'ils organisent.

Car il est aussi un autre enjeu majeur, plus directement économique, à la réception - le moment des départs est aussi un moment privilégié de remise «d'enveloppes», lesquelles passent de la main à la main lorsque l'invité-donateur s'apprête à partir. En effet, différentes catégories de parents cotisent lors de l'organisation de funérailles : enfants et beaux-enfants, petits-enfants adultes, neveux et nièces de la ou du défunt(e), frères et sœurs du conjoint endeuillé, etc. Le réseau social personnel des endeuillés intervient ensuite en seconde ligne, parfois également sous la forme de cotisations directement remises à l'intéressé, à l'initiative d'un groupe de collègues ou de coreligionnaires, par exemple. Les amis proches se manifestent aussi régulièrement, financièrement parlant, en amont des funérailles. Les remises d'enveloppes sont ensuite le fait du réseau social élargi : amis, collègues ou coreligionnaires ne s'étant pas entendu avec d'autres pour remettre une cotisation en amont, membres plus éloignés de la parentèle, et autres connaissances - la plus grande partie de l'assistance quittant toutefois les lieux sans avoir 
remis la moindre enveloppe. Les sommes glissées dans les enveloppes sont le plus souvent de 1000 ou 2000 francs $(1,5$ ou $3 €)$, mais peuvent aussi être bien supérieures dans les couches aisées de la population, et « une enveloppe de classe ", comme me le disait un interlocuteur régulier, "c'est à partir de 5000 francs ». Et surtout, une rumeur persistante suggère que certains modulent la somme qu' ils glissent dans l'enveloppe selon la façon dont ils ont été reçus - manière directe d'apprécier le sens des statuts et des hiérarchies manifesté par les endeuillés, et possible sanction économique immédiate du sentiment d'avoir été, ou pas, « minimisé »...

Le don d'enveloppes est en fait plein de calculs et, contrairement à ce que le recours à des enveloppes pourrait laisser penser, loin d'être anonyme. Car chacun(e) prend grand soin d'écrire son nom dans l'enveloppe qu'il remet, de manière à ce que l'endeuillé s'en souvienne lorsque les rôles seront inversés. Les endeuillés d'aujourd'hui sont en effet les invités de demain, et il n'est pas besoin d'être impeccablement "lettré» pour tenir un cahier reprenant les montants reçus lors des obsèques de ses parents et beaux-parents, afin de pouvoir « rendre» une somme adéquate le moment venu. Chacun(e) sait ainsi qu'il faut pouvoir se montrer solidaire de ceux qui ont manifesté leur solidarité lorsqu'on a soi-même été engagé dans l'organisation de funérailles. Mais on sait aussi à hauteur de quel montant telle ou telle personne s'est montrée solidaire, et le geste qu'on est prêt à poser tient largement compte de cette situation. Réciproquement d'ailleurs, les endeuillés anticipent les montants que tel ou telle devrait pouvoir leur remettre, et veillent à les recevoir en fonction.

$\mathrm{Au}$ final, c'est à une analyse multidimensionnelle des divisions sociales (Noret à paraître) qu'invitent les funérailles. La distinction de groupes de pairs, et de relations de patronage, sur la base du capital économique, lequel peut régulièrement être corrélé à un statut professionnel reconnu, constituent ainsi une première logique sociale forte dans la production de l'espace de la réception et de ses divisions. Employés et cadres du secteur public ou privé, mais aussi entrepreneurs et propriétaires de commerces détenteurs d'un registre de commerce, constituent ici globalement un pôle - lui-même bien entendu parcouru de lignes de divisions et hiérarchisé, avec à son sommet les « autorités » - auquel répond la masse des petits métiers du secteur dit « informel» (taxis-motos, menuisiers, maçons, vendeuses de nourriture, « revendeuses » d'ordres divers, etc.) et, occupant les régions les plus basses de l'espace social, les «paysans » et « villageois », parmi lesquels on trouve la quintessence des «pauvres ». Capital économique et statut professionnel constituent ainsi des forces sociales majeures dans la distribution spatiale des «invités », car ces propriétés sociales fonctionnent à deux niveaux : elles reflètent d'abord des écarts bien réels — «objectifs » — de conditions 
d'existence; elles jouent ensuite un rôle fondamental dans la production de classements sociaux où le capital économique fonctionne aussi largement comme capital symbolique.

Mais ces forces sociales ne sont pas seules, car l'univers de la parenté et des notabilités «traditionnelles » joue aussi un rôle structurant dans la production de l'espace de la réception : on cherche ainsi à gratifier les chefs des familles «parentes et alliées », même « pauvres » et « illettrés », de remerciements pour leur déplacement, d'une place et d'un repas dont ils ne devraient pas avoir à se plaindre. Et il en est de même des oncles et des tantes, tout « villageois » qu'ils puissent être. On le devine, les personnages capables de cumuler des formes de grandeur dans ces deux registres majeurs - celui de la surface économique et du statut professionnel d'une part; celui de la parenté et des notabilités « traditionnelles » d'autre part — sont évidemment ceux qui sont les plus susceptibles d'être reçus avec tous les égards dus à leur rang, tandis que ceux qui ne peuvent se réclamer ni de l'une, ni de l'autre forme de légitimité se dirigeront spontanément vers les lieux réservés au tout venant, et s'y trouveront parfaitement à leur place.

Il y aurait évidemment bien d'autres choses à écrire sur l'organisation des funérailles et des réceptions qui tout à la fois les clôturent et en constituent l'apothéose, mais cela nous emmènerait au-delà d'un texte comme celui-ci. Les pages qui précèdent permettent toutefois d'esquisser que, économodrames portés par les conduites d'honneur des endeuillé(e)s, les funérailles donnent à voir le Bénin d'aujourd'hui à travers l'un de ses lieux les plus massifs d'investissement collectif — lequel peut à bon droit être appréhendé comme révélateur de certaines dynamiques constitutives de la société en train de se faire.

Moments de nouage de rapports sociaux complexes, les funérailles africaines du milieu du $\mathrm{XX}^{\mathrm{e}}$ siècle auraient pu dès ce moment être érigées en « révélateurs » du changement social dans des sociétés en «reprise d'initiative» puis «en mutation». D'abord traitées toutefois par la recherche africaniste plutôt au prisme de leurs formes « ethniques », « coutumières » ou « traditionnelles », ce n'est finalement que plus tard qu'elles seront explorées dans leurs multiples dimensions, comme phénomènes susceptibles d'éclairer des modes plus généraux de déploiement du social, de ses dynamiques et de ses divisions.

Or, si Balandier n'a pas directement pris part au renouvellement des travaux sur les funérailles africaines qui se met en place à partir de la seconde 
partie des années 1980 (Vidal 1986; Gilbert 1988 ; Cohen \& Odhiambo 1992 ; Arhin 1994 ; Lentz 1994), il a certainement contribué à en inspirer les perspectives dans les travaux francophones. Mais plus fondamentalement, cet article a aussi cherché à montrer combien certaines des voies que Balandier a contribué à ouvrir ont conservé de leur pertinence pour penser l'Afrique « actuelle». Travailler avec Balandier, cela a été notamment pour moi, pouvoir construire son objet au travers d'un éclectisme méthodologique raisonné, en pouvant s'adosser à la fois à la sociologie et à l'anthropologie, au-delà des grands partages entre disciplines et entre sociétés, en faisant varier les échelles et les dispositifs d'enquête, en cherchant à porter une attention nuancée à la fois au poids du passé et aux aspirations au changement du présent.

Davantage que comme moments de restauration des sociétés - ce qui constitue l'héritage des travaux classiques, d'inspiration durkheimienne, sur le sujet - les funérailles ressortent d'un tel examen davantage comme révélateurs des dynamiques des collectifs, comme moment de leur mise à l'épreuve, de leur potentielle régénération comme de leur possible dislocation. Occasions de mise en jeu du « potentiel social de chacun » (Vidal 1986:15), elles constituent des occasions de dépense sociale investies à la hauteur des enjeux protéiformes qu'y discernent les acteurs, et peuvent à cet égard être considérées comme des sites productifs d'observation des dynamiques de l'espace social.

Laboratoire d'Anthropologie des Mondes Contemporains, Université libre de Bruxelles, Belgique.

BIBLIOGRAPHIE

ARHIN K., 1994, « The Economic Implications of Transformations in Akan Funeral Rites », Africa, 64 (3) : 307-322.

Balandier G., 1970 (DIR.), Sociologie des mutations, Paris, Éditions Anthropos.

- 1982 [1955], Sociologie actuelle de l'Afrique noire: dynamique sociale en Afrique centrale, Paris, PUF.

— 1985a [1955], Sociologie des Brazzavilles noires, Paris, Presses de la Fondation nationale de Sciences politiques. 
— 1985b [1974], Anthropo-logiques, Paris, Le Livre de Poche.

— 1991, «Préface », in C. VIDAL, Sociologie des passions, Paris, Karthala : 5-8.

— 2004 [1971], Sens et puissance. Les dynamiques sociales, Paris, PUF.

— 2008 [1957], Afrique ambiguë, Paris, Plon.

Bourdieu P., 1984, "Espace social et genèse des "classes" ", Actes de la recherche en sciences sociales, 52-53:3-14.

Cohen D. W. \& Odhiambo E. S. A., 1992, Burying SM. The Politics of Knowledge and the Sociology of Power in Africa, Oxford, James Currey.

Droz Y., 2011, « Transformations of Death among the Kikuyu of Kenya : From Hyenas to Tombs », in M. JINDRA \& J. NORET (eds.), Funerals in Africa. Explorations of a Social Phenomenon, New York-London, Berghahn : 69-87.

DuRKheIm E., 1994 [1912], Les formes élémentaires de la vie religieuse, Paris, PUF.

ForTes M., 1961, "Pietas in Ancestor Worship », Journal of the Royal Anthropological Institute, 91 (2) : 166-191.

VAN DER GeEst S., 2006, « Between Death and Funeral : Mortuaries and the Exploitation of Liminality in Kwahu, Ghana », Africa, 76 (4) : 485-501.

Gilbert M., 1988, « The Sudden Death of a Millionaire : Conversion and Consensus in a Ghanaian Kingdom », Africa, 58 (3) : 291-314.

Goody J., 1962, Death, Property, and the Ancestors. A Study of the Mortuary Customs of the LoDagaa of West Africa, London, Tavistock Publications.

GlucKman M., 1940, «Analysis of a Social Situation in Modern Zululand », Bantu Studies, 14 (1) : 1-30.

— 1947, « Malinowski's "Functional” Analysis of Social Change », Africa, 17 (2) : 103-121.

HERTz R., 1970 [1907], « Contribution à une étude sur la représentation collective de la mort », in R. HerTz, Sociologie religieuse et folklore, Paris, PUF : 1-83.

JACKSON M., 1977, «The Identity of the Dead. Aspects of Mortuary Ritual in a West African Society », Cahiers d'Etudes africaines, XVII (2-3), 66-67: 271-297.

JINDRA M., 2005, « Christianity and the Proliferation of Ancestors : Changes in Hierarchy and Mortuary Ritual in the Cameroon Grassfields », Africa, 75 (3) : 356-377.

JiNDRA M. \& NoRET J., 2011, «African Funerals and Sociocultural Change », in M. JINDRA \& J. Noret (eds.), Funerals in Africa. Explorations of a Social Phenomenon, New York-London, Berghahn : 16-40. 
LAmont M., 2011, « Decomposing Pollution? Corpses, Burials, and Affliction among the Meru of Central Kenya », in M. Jindra \& J. Noret (eds.), Funerals in Africa. Explorations of a Social Phenomenon, New York-London, Berghahn : 88-108.

Lemay P., Noret J. \& Somville V., 2013, «Facing Misfortune : Expenditures on MagicoReligious Powers for Cure and Protection in Benin », Journal of African Economies, $22(2): 300-322$.

Lentz C., 1994, « Home, Death and Leadership : Discourses of an Educated Elite from North-Western Ghana », Social Anthropology, 2 (2) : 149-169.

DE L'Estolle B., 1994, «L'anthropologue face au monde moderne. Malinowski et la rationalisation de l'anthropologie et de l'administration », Genèses, 17 (1) : 140-163.

Little K., 1953, " The Study of "Social Change" in British West Africa », Africa, 23 (4) : 274-284.

MAIR L., 1934, «The Study of Culture Contact as a Practical Problem », Africa, 7 (4): 415-422.

Malinowski B., 1929, «Practical Anthropology », Africa, 2 (1) : 22-38.

- 1935, Coral Gardens and their Magic, vol. 1, London, Routledge.

— 1945, The Dynamics of Culture Change, New Haven, Yale University Press.

- 1954 [1925], Magic, Science, and Religion, and Other Essays, New York, Anchor Books.

— 1984 [1922], Argonauts of the Western Pacific, Long Grove, Waveland Press.

MARY A., 2017, «Ethnographie de soi sous le “zéro équatorial”. Le chantier autobiographique de Georges Balandier », L'Homme, 221 : 11-40.

Middleton J., 1982, «Lugbara Death », in M. Bloch \& J. PARry (eds.), Death and the Regeneration of Life, Cambridge, Cambridge University Press : 134-154.

Mitchell J. C., 1956, The Kalela Dance. Aspects of Social Relationships among Urban Africans in Northern Rhodesia, Manchester, Manchester University Press.

Morton-Williams P., 1960, "Yoruba Responses to the Fear of Death », Africa, 30 (1): $34-40$.

Noret J., 2004, « Morgues et prise en charge de la mort au Sud-Bénin », Cahiers d'Études africaines, XLIV (4), 176 : 745-767.

—2006, Autour de «ceux qui n'existent plus ». Deuil, funérailles et place des défunts au Sud-Bénin, Thèse de doctorat, Bruxelles, ULB ; Paris, EHESS.

— 2010, Deuil et funérailles dans le Bénin méridional. Enterrer à tout prix, Bruxelles, Éditions de l'Université de Bruxelles. 
— 2012, «Grief as Social Fact. Notes from Southern Benin », African Studies, 71 (2): 273-286.

— 2014 «Sur le dos des morts ? Organiser des funérailles catholiques à Abomey », Terrain, 62: 54-69.

À paraître, «For a Multidimensional Class Analysis in Africa », Review of African Political Economy.

Onwuzuruigbo I., 2014, «Space of Power and the Power of Space. Islam and Conflict over Mortuary Space in Colonial Ibadan, Journal of Urban History, 40 (2) : 301-317.

OtTEnBerg S., 1955, «Improvement Associations among the Afikpo Ibo », Africa, 25 (1) : $1-28$.

Page B., 2007, « Slow-Going: The Mortuary, Modernity, and the Hometown Association in Bali-Nyonga ", Africa, 77 (3) : 419-441.

Palgi P. \& Abramovitch H., 1984, «Death : A Cross-Cultural Perspective », Annual Review of Anthropology, $13: 385-417$.

PARker J., 2000, « The Cultural Politics of Death and Burial in Early Colonial Accra », in D. ANDERSON \& R. RATHBone (eds.), Africa's Urban Past, Oxford, James Currey : 205-221.

Radcliffe-Brown A. R., 1922, The Andaman Islanders. A Study in Social Anthropology, Cambridge, Cambridge University Press.

RAnger T., 2004, « Dignifying Death : the Politics of Burial in Bulawayo », Journal of Religion in Africa, 34 (1-2) : 110-144.

Thomas L.-V., 1960, «Un système philosophique sénégalais : la cosmologie des Diola », Présence Africaine, 32-33 : 64-76.

— 1995, «Leçon pour l'Occident : ritualité du chagrin et du deuil en Afrique noire », in T. Nathan et al. (dir.), Rituels de deuil, travail du deuil, Paris, La Pensée Sauvage : $17-65$.

— 2006, «Eléments pour un itinéraire», Sociétés, 93 : 39-43.

Tonda J., 2000, «Enjeux du deuil et négociation des rapports sociaux de sexe au Congo », Cahiers d'Études africaines, XL (1), 157 : 5-24.

VAn Gennep A., 1981 [1909], Les rites de passage, Paris, Picard.

VIDAL C., 1986, «Funérailles et conflit social en Côte d'Ivoire », Politique Africaine, $24: 9-19$.

— 1991, Sociologie des passions (Côte d'Tvoire, Rwanda), Paris, Karthala.

Wilson G., 1939, « Nyakyusa Conventions of Burial », Bantu Studies, 13 (1) : 1-31. 


\title{
RÉSUMÉ
}

Ce texte est organisé en deux parties. La première partie remet en perspective historique la rencontre manquée entre les travaux portant sur le changement social africain, dans lesquels va s'inscrire Balandier à partir des années 1950, et la dynamique historique des funérailles africaines de la même période. La seconde partie revient sur l'actualité de l'héritage de Balandier pour penser les dynamiques contemporaines des funérailles africaines, lesquelles sont considérées ici comme des points d'entrée féconds à partir desquels il est possible d'explorer avec profit des dynamiques plus générales du champ social.

Mots-clés: Bénin, Balandier, changement social, espace social, funérailles, mort.

\begin{abstract}
Current Sociology of Funerals. The Management of Death and the Social Space in Contemporary Africa. - This article is organized in two parts. The first half of the text is an attempt at understanding the missed encounter between, on the one hand, the corpus of literature emerging on topics related to African social change since the 1930s - that is, the corpus of literature to which Balandier contributed from the 1950s onwards - and, on the other, the historical dynamics of African funerals during the colonial period. The second part of the text then goes on to argue for the relevance of Georges Balandier's intellectual heritage in exploring the current dynamics of African funerals, which are here considered as productive in highlighting more generalized dynamics of the social field.
\end{abstract}

Keywords: Benin, Balandier, death, funerals, social change, social space. 